\title{
Invariance principles for hybrid systems with memory
}

\author{
Jun Liu ${ }^{\mathrm{a}, *}$, Andrew R. Teel ${ }^{\mathrm{b}}$ \\ ${ }^{a}$ Department of Automatic Control and Systems Engineering, University of Sheffield, Sheffield S1 3JD, United Kingdom \\ ${ }^{b}$ Department of Electrical and Computer Engineering, University of California, Santa Barbara, CA 93106, USA
}

\begin{abstract}
Hybrid systems with memory are dynamical systems exhibiting both delayed and hybrid dynamics. Such systems can be described by hybrid functional inclusions. Classical invariance principles play an instrumental role in proving stability and convergence of dynamical systems. Invariance principles for general hybrid systems with delays, however, remain an open topic. In this paper, we prove invariance principles for hybrid systems with memory, using both Lyapunov-Razumikhin function and Lyapunov-Krasovskii functional methods. These invariance principles are then applied to derive two stability results as corollaries.
\end{abstract}

Keywords:

Hybrid systems with memory, hybrid functional inclusions, invariance principles, stability.

\section{Introduction}

Hybrid systems with memory refer to a class of dynamical systems exhibiting both delayed and hybrid dynamics. Such systems naturally occur, e.g. in control applications where hybrid control algorithms are used with the presence of delays in the control loop, and have attracted considerable attention in the past decade (see, e.g., [3, 12, 13, 18, 19, 24-26]). Classical invariance principles [9, 10] play an instrumental role in proving stability and convergence properties of dynamical systems. As such, they have been extended by various authors to hybrid dynamical systems (e.g., $[1,2,4,8,11,12,20,22,23])$ and time-delay systems (e.g., $[6,7,12])$.

Invariance principles for general hybrid systems with delays, however, remain an open topic. The main difficulty in establishing such results lies in proving certain invariance properties of the limit set of memories resulted from a hybrid trajectory. For ordinary differential equations or hybrid systems without delays, the limit set involves no memory and is a subset of the Euclidean space. For delay differential equations, such memories are always encoded in continuous functions and the notion of uniform convergence topology is well-suited for studying their limits. The memory for hybrid systems with delays, however, could include discontinuities caused by jumps. Consequently, the uniform convergence topology is no longer appropriate for analyzing the limit behaviors of such systems.

Recent work in [14, 16] introduces a framework for studying hybrid systems with delays through generalized solutions. To overcome the difficulty in handling discontinuities caused by jumps in hybrid systems with delays, the results in $[14,16]$ rely on a phase space of hybrid memory arcs equipped with the graphical convergence topology, instead of a space of piecewise continuous functions equipped with the conventional uniform convergence topology. By using tools from functional differential inclusions, basic existence

\footnotetext{
*Corresponding author. Tel.: (+44) (0)114 2225632.

Email addresses: j.liu@sheffield.ac.uk (Jun Liu), teel@ece.ucsb.edu (Andrew R. Teel)
} 
and well-posedness results have been established [15]. Moreover, stability results using both LyapunovRazumikhin function and Lyapunov-Krasovskii functional methods have also been proved [16, 17].

The main contributions of this paper include two new invariance principles established for hybrid systems with memory. This work was made possible by applying the notion of graphical convergence of hybrid memory arcs along the line of work in $[14,16]$. As main applications of these new invariance principles, we show that two stability results for hybrid systems with memory can be derived as immediate corollaries.

The rest of this paper is organized as follows. In Section 2, we present some preliminaries for hybrid systems with memory, mainly taken from [15], and prove a basic proposition that concludes weak invariance of the limit set, in graphical convergence sense, of the memories resulted from a hybrid trajectory. Sections 3 include the main results on invariance principles and Section 4 illustrate two main applications of the invariance principles in the stability analysis of hybrid systems with memory. We illustrate the stability results with a simple example in Section 5 and conclude the paper in Section 6.

\section{Preliminaries}

Notation: $\mathbb{R}^{n}$ denotes the $n$-dimensional Euclidean space with its norm denoted by $|\cdot| ; \mathbb{Z}$ denotes the set of all integers; $\mathbb{R}_{\geq 0}=[0, \infty), \mathbb{R}_{\leq 0}=(-\infty, 0], \mathbb{Z}_{\geq 0}=\{0,1,2, \cdots\}$, and $\mathbb{Z}_{\leq 0}=\{0,-1,-2, \cdots\}$.

\subsection{Hybrid systems with memory}

Definition 2.1 ([15]). Consider a subset $E \subseteq \mathbb{R} \times \mathbb{Z}$ with $E=E_{\geq 0} \cup E_{\leq 0}$, where $E_{\geq 0}:=\left(\mathbb{R}_{\geq 0} \times \mathbb{Z}_{\geq 0}\right) \cap E$ and $E_{\leq 0}:=\left(\mathbb{R}_{\leq 0} \times \mathbb{Z}_{\leq 0}\right) \cap E$. It is called a compact hybrid time domain with memory if

$$
E_{\geq 0}=\bigcup_{j=0}^{J-1}\left(\left[t_{j}, t_{j+1}\right], j\right)
$$

and

$$
E_{\leq 0}=\bigcup_{k=1}^{K}\left(\left[s_{k}, s_{k-1}\right],-k+1\right)
$$

for some finite sequence of times $s_{K} \leq \cdots \leq s_{1} \leq s_{0}=0=t_{0} \leq t_{1} \leq \cdots \leq t_{J}$. It is called a hybrid time domain with memory if, for all $(T, J) \in E_{\geq 0}$ and all $(S, K) \in \mathbb{R}_{\geq 0} \times \mathbb{Z}_{\geq 0},\left(E_{\geq 0} \cap([0, T] \times\{0,1, \cdots, J\})\right) \cup$ $\left(E_{\leq 0} \cap([-S, 0] \times\{-K,-K+1, \cdots, 0\})\right)$ is a compact hybrid time domain with memory. The set $E_{\leq 0}$ is called a hybrid memory domain.

Definition 2.2 ([15]). A hybrid arc with memory consists of a hybrid time domain with memory, denoted by $\operatorname{dom} x$, and a function $x: \operatorname{dom} x \rightarrow \mathbb{R}^{n}$ such that $x(\cdot, j)$ is locally absolutely continuous on $I^{j}=$ $\{t:(t, j) \in \operatorname{dom} x\}$ for each $j \in \mathbb{Z}$ such that $I^{j}$ has nonempty interior. In particular, a hybrid arc $x$ with memory is called a hybrid memory arc if $\operatorname{dom} x \subseteq \mathbb{R}_{\leq 0} \times \mathbb{Z}_{\leq 0}$. We shall simply use the term hybrid arc if we do not have to distinguish between the above two hybrid arcs. We write $\operatorname{dom} \geq 0(x):=\operatorname{dom} x \cap\left(\mathbb{R}_{\geq 0} \times \mathbb{Z}_{\geq 0}\right)$ and $\operatorname{dom}_{\leq 0}(x):=\operatorname{dom} x \cap\left(\mathbb{R}_{\leq 0} \times \mathbb{Z}_{\leq 0}\right)$.

We shall use $\mathcal{M}$ to denote the collection of all hybrid memory arcs. Moreover, given $\Delta \in[0, \infty)$, we denote by $\mathcal{M}^{\Delta}$ the collection of hybrid memory arcs $\varphi$ satisfying the following two conditions: (1) $s+k \geq$ $-\Delta-1$ for all $(s, k) \in \operatorname{dom} \varphi$; and (2) there exists $\left(s^{\prime}, k^{\prime}\right) \in \operatorname{dom} \varphi$ such that $s^{\prime}+k^{\prime} \leq-\Delta$.

Given a hybrid arc $x$, we define an operator $\mathcal{A}_{[,,]}^{\Delta} x: \operatorname{dom}_{\geq 0}(x) \rightarrow \mathcal{M}^{\Delta}$ by

$$
\mathcal{A}_{[t, j]}^{\Delta} x(s, k)=x(t+s, j+k),
$$


for all $(s, k) \in \operatorname{dom}\left(\mathcal{A}_{[t, j]}^{\Delta} x\right)$, where

$$
\operatorname{dom}\left(\mathcal{A}_{[t, j]}^{\Delta} x\right):=\left\{(s, k) \in \mathbb{R}_{\leq 0} \times \mathbb{Z}_{\leq 0}:(t+s, j+k) \in \operatorname{dom} x, s+k \geq-\Delta_{\text {inf }}\right\},
$$

where

$$
\Delta_{\text {inf }}:=\inf \{\delta \geq \Delta: \exists(t+s, j+k) \in \operatorname{dom} x \text { s.t. } s+k=-\delta\} .
$$

It follows that if $\mathcal{A}_{[0,0]}^{\Delta} x \in \mathcal{M}^{\Delta}$, then $\mathcal{A}_{[t, j]}^{\Delta} x \in \mathcal{M}^{\Delta}$ for any $(t, j) \in \operatorname{dom} \geq 0(x)$.

Definition 2.3. A hybrid system with memory of size $\Delta$ is defined by a 4-tuple $\mathcal{H}_{\mathcal{M}}^{\Delta}=(\mathcal{C}, \mathcal{F}, \mathcal{D}, \mathcal{G})$ :

- a set $\mathcal{C} \subseteq \mathcal{M}^{\Delta}$, called the flow set;

- a set-valued functional $\mathcal{F}: \mathcal{M}^{\Delta} \rightrightarrows \mathbb{R}^{n}$, called the flow map;

- a set $\mathcal{D} \subseteq \mathcal{M}^{\Delta}$, called the jump set;

- a set-valued functional $\mathcal{G}: \mathcal{M}^{\Delta} \rightrightarrows \mathbb{R}^{n}$, called the jump map.

Definition 2.4. A hybrid arc is a solution to the hybrid system $\mathcal{H}_{\mathcal{M}}^{\Delta}$ if $\mathcal{A}_{[0,0]}^{\Delta} x \in \mathcal{C} \cup \mathcal{D}$ and:

(S1) for all $j \in \mathbb{Z}_{\geq 0}$ and almost all $t$ such that $(t, j) \in \operatorname{dom}_{\geq 0}(x)$,

$$
\mathcal{A}_{[t, j]}^{\Delta} x \in \mathcal{C}, \quad \dot{x}(t, j) \in \mathcal{F}\left(\mathcal{A}_{[t, j]}^{\Delta} x\right),
$$

(S2) for all $(t, j) \in \operatorname{dom}_{\geq 0}(x)$ such that $(t, j+1) \in \operatorname{dom}_{\geq 0}(x)$,

$$
\mathcal{A}_{[t, j]}^{\Delta} x \in \mathcal{D}, \quad x(t, j+1) \in \mathcal{G}\left(\mathcal{A}_{[t, j]}^{\Delta} x\right) .
$$

The solution $x$ is called nontrivial if $\operatorname{dom}_{\geq 0}(x)$ has at least two points. It is called complete if $\operatorname{dom}_{\geq 0}(x)$ is unbounded. It is called maximal if there does not exist another solution $y$ to $\mathcal{H}_{\mathcal{M}}^{\Delta}$ such that dom $x$ is a proper subset of dom $y$ and $x(t, j)=y(t, j)$ for all $(t, j) \in \operatorname{dom} x$. The set of all maximal solutions to $\mathcal{H}_{\mathcal{M}}^{\Delta}$ is denoted by $\mathcal{S}_{\mathcal{H}_{\mathcal{M}}^{\Delta}}$.

\subsection{Set convergence and graphical distance}

The following notion of set convergence is taken from [21, Chapter 4].

Definition 2.5 (Set convergence). Consider a sequence of sets $\left\{H_{i}\right\}_{i=1}^{\infty}$ in $\mathbb{R}^{n}$. The outer limit of the sequence, denoted by $\lim \sup _{i \rightarrow \infty} H_{i}$ is the set of all $x \in \mathbb{R}^{n}$ for which there exists a subsequence $x_{i_{k}} \in S_{i_{k}}, k=1,2, \cdots$, such that $x_{i_{k}} \rightarrow x$. The inner limit of $\left\{H_{i}\right\}_{i=1}^{\infty}$, denoted by $\liminf _{i \rightarrow \infty} H_{i}$, is the set of all $x \in \mathbb{R}^{n}$ for which there exists a sequence $x_{i} \in S_{i}$ such that $x_{i} \rightarrow x$. The limit of $\left\{H_{i}\right\}_{i=1}^{\infty}$ exists if lim sup $\operatorname{sim}_{i \rightarrow \infty} H_{i}=\liminf _{i \rightarrow \infty} H_{i}$ and it is then given by $\lim _{i \rightarrow \infty} H_{i}=\limsup _{i \rightarrow \infty} H_{i}=\liminf _{i \rightarrow \infty} H_{i}$.

We now focus on hybrid memory $\operatorname{arcs}$ in $\mathcal{M}^{\Delta}$. Note that $\mathcal{M}^{\Delta}$ is not a vector space, since different hybrid arcs may have different domains. We recall from [21] a quantity that characterizes the set convergence of closed nonempty sets and use this distance to define a metric on $\mathcal{M}^{\Delta}$.

Let cl-sets $\neq \varnothing\left(\mathbb{R}^{n}\right)$ denote the collection of all nonempty, closed subsets of $\mathbb{R}^{n}$. Given $\rho \geq 0$, for each pair $A, B \in$ cl-sets $_{\equiv \varnothing \varnothing}\left(\mathbb{R}^{n}\right)$, define

$$
\mathbf{d}_{\rho}(A, B):=\max _{|z| \leq \rho}|d(z, A)-d(z, B)| .
$$

where $d(z, H)$ for $z \in \mathbb{R}^{n}$ and $H \subseteq \mathbb{R}^{n}$ is defined by $\inf _{w \in H}|w-z|$. Furthermore, define

$$
\mathbf{d}(A, B):=\int_{0}^{\infty} \mathbf{d}_{\rho}(A, B) e^{-\rho} d \rho,
$$

which is called the (integrated) set distance between $A$ and $B$. This distance indeed characterizes set convergence of sets in cl-sets $\neq \varnothing\left(\mathbb{R}^{n}\right)$ as recalled below. 
Theorem 2.1. [21, Theorem 4.42] A sequence $S_{i} \in c l$-sets $\varnothing \varnothing\left(\mathbb{R}^{n}\right)$ converges to $S$ if and only $\mathbf{d}\left(S_{i}, S\right) \rightarrow 0$. Moreover, the space $\left(c l\right.$-sets $\left.\neq \varnothing\left(\mathbb{R}^{n}\right), \mathbf{d}\right)$ is a separable, locally compact, and complete metric space.

Consider the following subspaces of $\left(\mathcal{M}^{\Delta}, \mathbf{d}\right)$ for some $b, \lambda \geq 0$ :

$$
\begin{aligned}
\mathcal{M}_{b}^{\Delta} & :=\left\{\varphi \in \mathcal{M}^{\Delta}:\|\varphi\|:=\sup _{(s, k) \in \operatorname{dom} \varphi}|\varphi(s, k)| \leq b\right\}, \\
\mathcal{M}_{b, \lambda}^{\Delta}: & =\left\{\varphi \in \mathcal{M}_{b}^{\Delta}: \varphi \text { is } \lambda \text {-Lipschitz }\right\},
\end{aligned}
$$

where $\varphi \in \mathcal{M}$ is said to be $\lambda$-Lipschitz if $\left|\varphi(s, k)-\psi\left(s^{\prime}, k\right)\right| \leq \lambda\left|s-s^{\prime}\right|$ holds for all $(s, k),\left(s^{\prime}, k\right) \in \operatorname{dom} \varphi$. The following proposition is proved in [15] as a corollary of Theorem 2.1.

Proposition 2.1. [15] The space $\left(\mathcal{M}_{b, \lambda}^{\Delta}, \mathbf{d}\right)$ is a separable, locally compact, and complete metric space.

Definition 2.6. Given some $\varphi \in \mathcal{M}^{\Delta}$, a sequence $\left\{\varphi_{i}\right\}_{i=1}^{\infty} \subseteq \mathcal{M}^{\Delta}$, and a set $\mathcal{R} \subseteq \mathcal{M}^{\Delta}$,

- we write $\varphi_{i} \stackrel{\text { gph }}{\longrightarrow} \varphi$ as $i \rightarrow \infty$ or $\lim \operatorname{gph}_{i \rightarrow \infty} \varphi_{i}=\varphi$, if $\mathbf{d}\left(\varphi_{i}, \varphi\right) \rightarrow 0$ as $i \rightarrow \infty$.

- we write $\varphi_{i} \stackrel{\text { gph }}{\longrightarrow} \mathcal{R}$ as $i \rightarrow \infty$, if $\lim _{i \rightarrow \infty} \operatorname{dist}\left(\varphi_{i}, \mathcal{R}\right)=0$, where

$$
\operatorname{dist}(\psi, \mathcal{R}):=\inf _{\chi \in \mathcal{R}} \mathbf{d}(\chi, \psi),
$$

for $\psi \in \mathcal{R}$.

One of the main motivations of considering graphical convergence, instead of uniform convergence, for hybrid memory arcs is that graphical convergence is more suitable for handling the jumps in hybrid memory arcs, as illustrated by the following simple example.

Example 2.1. Consider a sequence of hybrid memory arcs $\left\{\varphi_{i}\right\}_{i=1}^{\infty}$ in $\mathcal{M}^{\Delta}$ given by $\varphi_{i}(s, 0) \equiv 1$ and $\varphi_{i}(s,-1) \equiv 0$, where $\operatorname{dom} \varphi_{i}=\left(\left[t_{i}, 0\right] \times\{0\}\right) \cup\left(\left[-\Delta, t_{i}\right] \times\{-1\}\right)$ and $\left\{t_{i}\right\}_{i=1}^{\infty}$ is a strictly increasing sequence such that $t_{i} \rightarrow t \in[-\Delta, 0]$ as $i \rightarrow \infty$. Consider another hybrid memory arc $\varphi$ in $\mathcal{M}^{\Delta}$ defined by $\varphi(s, 0) \equiv 1$ and $\varphi(s,-1) \equiv 0$, where $\operatorname{dom} \varphi=([t, 0] \times\{0\}) \cup([-\Delta, t] \times\{-1\})$. It is easy to verify that $\varphi_{i} \stackrel{\text { gph }}{\longrightarrow} \varphi$ as $i \rightarrow \infty$. However, since $\varphi_{i}$ are $\varphi$ have different domains, we cannot compare their distance directly. If we were to define the uniform distance between $\varphi_{i}$ and $\varphi$ by the quality

$$
\mathbf{d}_{\text {uniform }}\left(\varphi_{i}, \varphi\right):=\sup _{\substack{(s, k) \in \operatorname{dom} \varphi \\(s, j) \in \operatorname{dom} \varphi_{i}}}\left|\varphi_{i}(s, j)-\varphi(s, k)\right|,
$$

we then have $\mathbf{d}_{\text {uniform }}\left(\varphi_{i}, \varphi\right)=1$ all $i \geq 1$. In other words, the sequence of hybrid memory arcs $\left\{\varphi_{i}\right\}_{i=1}^{\infty}$ would not converge to $\varphi$ in this sense.

\subsection{Nominal well-posedness}

A standing assumption of this paper is that a hybrid system with memory is nominally well-posed, which is a special case of well-posedness considered in [15].

Definition 2.7 (Nominal well-posedness of hybrid systems with memory [14]). A hybrid system $\mathcal{H}_{\mathcal{M}}^{\Delta}$ is said to be nominally well-posed if the following properties hold: for every graphically convergent sequence $\left\{x_{i}\right\}_{i=1}^{\infty}$ of solutions to $\mathcal{H}_{\mathcal{M}}^{\Delta}$ with $\mathcal{A}_{[0,0]}^{\Delta} x_{i} \in \mathcal{M}_{b, \lambda}^{\Delta}$ for some $b, \lambda \geq 0$ and $\mathcal{A}_{[0,0]}^{\Delta} x_{i} \stackrel{\text { gph }}{\longrightarrow} \varphi \in \mathcal{M}^{\Delta}$,

(a) if the sequence $\left\{x_{i}\right\}_{i=1}^{\infty}$ is locally eventually bounded (that is, for any $m>0$, there exists $N>0$ and $k>0$ such that, for all $i>N$ and all $(t, j) \in \operatorname{dom} x_{i}$ with $\left.t+j<m,\left|x_{i}(t, j)\right|<k\right)$, then its graphical limit $x$ is a solution to $\mathcal{H}_{\mathcal{M}}^{\Delta}$ with $\mathcal{A}_{[0,0]}^{\Delta} x=\varphi$ and $\operatorname{dom} x=\lim _{i \rightarrow \infty} \operatorname{dom} x_{i}$; 
(b) if the sequence $\left\{x_{i}\right\}_{i=1}^{\infty}$ is not locally eventually bounded, then there exist some $T, J \in(0, \infty)$ and a sequence $\left\{t_{i}\right\}_{i=1}^{\infty}$ with $\left(t_{i}, J\right) \in \operatorname{dom} x_{i}$ for sufficiently large $i$ such that $\lim _{i \rightarrow \infty} t_{i}=T, \lim _{i \rightarrow \infty}\left|x_{i}\left(t_{i}, J\right)\right|=$ $\infty$, and the limit $x=\operatorname{limgph}_{i \rightarrow \infty} x_{i}$ restricted to the domain $\{(t, j) \in \operatorname{dom} x: t+j<T+J\}$ is a maximal solution to $\mathcal{H}_{\mathcal{M}}^{\Delta}$ with $\mathcal{A}_{[0,0]}^{\Delta} x=\varphi$ and $\lim _{t \rightarrow T}|x(t, J)|=\infty$.

The following is a list of basic conditions on the data of the hybrid system $\mathcal{H}_{\mathcal{M}}^{\Delta}=(\mathcal{C}, \mathcal{F}, \mathcal{D}, \mathcal{G})$, recalled from [15].

Assumption 2.1 ([15]). For every $b, \lambda \geq 0$, the following hold:

(A1) $\mathcal{C} \cap \mathcal{M}_{b, \lambda}^{\Delta}$ and $\mathcal{D} \cap \mathcal{M}_{b, \lambda}^{\Delta}$ are closed subsets of $\mathcal{M}^{\Delta}$;

(A2) $\mathcal{F}$ is outer semicontinuous relative to the set $\mathcal{C} \cap \mathcal{M}_{b, \lambda}^{\Delta}$, locally bounded relative to the set $\mathcal{C} \cap \mathcal{M}_{b}^{\Delta}$, and $\mathcal{F}(\varphi)$ is nonempty and convex for each $\varphi \in \mathcal{C} \cap \mathcal{M}_{b, \lambda}^{\Delta}$;

(A3) $\mathcal{G}$ is outer semicontinuous relative to the set $\mathcal{D} \cap \mathcal{M}_{b, \lambda}^{\Delta}$, locally bounded relative to the set $\mathcal{D} \cap \mathcal{M}_{b}^{\Delta}$, and $\mathcal{G}(\varphi)$ is nonempty for each $\varphi \in \mathcal{D} \cap \mathcal{M}_{b, \lambda}^{\Delta}$.

The following result first appeared in [14] and the proof was included in [15] as a special case of wellposedness.

Theorem $2.2([14,15])$. If a hybrid system with memory $\mathcal{H}_{\mathcal{M}}^{\Delta}=(\mathcal{C}, \mathcal{F}, \mathcal{D}, \mathcal{G})$ satisfies Assumption 2.1, then it is nominally well-posed.

The main focus of this paper is on invariance principles for hybrid systems with memory. The readers are referred to [14]-[15] for existence of generalized solutions and well-posedness for hybrid systems with memory. Some preliminaries on invariance and limit set for hybrid systems with memory are given next.

\subsection{Limit set and weak invariance}

Now we are ready to present and prove some preliminary results on invariance properties of the limit set of a pre-compact (i.e., bounded and complete) solution to $\mathcal{H}_{\mathcal{M}}^{\Delta}$ in terms of graphical convergence.

Definition 2.8. The $\omega$-limit set of a hybrid arc with memory $x: \operatorname{dom} \varphi \rightarrow \mathbb{R}^{n}$, denoted by $\Omega(x)$, is the set of all hybrid memory arcs $\varphi \in \mathcal{M}^{\Delta}$ for which there exists a sequence of pairs $\left\{\left(t_{i}, j_{i}\right)\right\}_{i=1}^{\infty}$ such that $\left(t_{i}, j_{i}\right) \in \operatorname{dom} x, t_{i}+j_{i} \rightarrow \infty$ and $\mathcal{A}_{\left[t_{i}, j_{i}\right]}^{\Delta} \stackrel{\text { gph }}{\longrightarrow} \varphi$ as $i \rightarrow \infty$.

Definition 2.9. Given a hybrid system with memory $\mathcal{H}_{\mathcal{M}}^{\Delta}$, a set $\mathcal{S} \subseteq \mathcal{M}^{\Delta}$ is said to be weakly positively invariant if, for every $\varphi \in \mathcal{S}$, there exists at least one complete solution $x \in \mathcal{S}_{\mathcal{H}_{\mathcal{M}}^{\Delta}}$ with $\mathcal{A}_{[0,0]}^{\Delta} x=\varphi$ such that $\mathcal{A}_{[t, j]}^{\Delta} x \in \mathcal{S}$ for all $(t, j) \in \operatorname{dom}_{\geq 0}(x)$.

Proposition 2.2. Let $x$ be a solution for $\mathcal{H}_{\mathcal{M}}^{\Delta}$ that is bounded and complete. Then $\Omega(x) \subseteq \mathcal{M}_{b, \lambda}^{\Delta}$ for some $b, \lambda>0$ and it is weakly positively invariant and $\mathcal{A}_{[t, j]}^{\Delta} \stackrel{g p h}{\longrightarrow} \Omega(x)$ as $t+j \rightarrow \infty$ and $(t, j) \in \operatorname{dom}_{\geq 0}(x)$.

To prove this proposition, the following lemma, recalled from [15], is used.

Lemma 2.1. Let $\left\{z_{i}\right\}_{i=1}^{\infty}$ be a graphically convergent sequence of solutions to $\mathcal{H}_{\mathcal{M}}^{\Delta}$ with limit $z$. If $\left\{z_{i}\right\}_{i=1}^{\infty}$ is locally eventually bounded, then the following hold:

1. $\left\{\mathcal{A}_{[t, j]}^{\Delta} z_{i}\right\}_{i=1}^{\infty}$ graphically converges to $\mathcal{A}_{[t, j]}^{\Delta} z$ for each $t \in \operatorname{int}\left(I_{j}\right)$;

2. if $(t, j) \in \operatorname{dom} z$ and $(t, j+1) \in$ dom $z$, there exists a sequence $\left\{s_{i}\right\}_{i=1}^{\infty}$ such that the following hold simultaneously: $\left(s_{i}, j\right) \in \operatorname{dom}\left(z_{i}\right),\left(s_{i}, j+1\right) \in \operatorname{dom}\left(z_{i}\right), \lim _{i \rightarrow \infty} s_{i}=t, \lim _{i \rightarrow \infty} z_{i}\left(s_{i}, j\right)=z(t, j), \lim _{i \rightarrow \infty} z_{i}\left(s_{i}, j+\right.$ $1)=z(t, j+1), \lim g p h_{i \rightarrow \infty} \mathcal{A}_{\left[s_{i, j}\right]}^{\Delta} z_{i}=\mathcal{A}_{[t, j]}^{\Delta} z$, and limgph $h_{i \rightarrow \infty} \mathcal{A}_{\left[s_{i, j+1}\right]}^{\Delta} z_{i}=\mathcal{A}_{\left[s_{i, j}, 1\right]}^{\Delta} z$. 
Proof of Proposition 2.2. The fact that $\Omega(x) \subseteq \mathcal{M}_{b, \lambda}^{\Delta}$ for some $b, \lambda>0$ follows from the boundedness assumptions on $x$ and $\mathcal{F}$ and Proposition 2.1.

Pick any $\varphi \in \Omega(x)$. By the definition of $\Omega(x)$, there exists a sequence of pairs $\left\{\left(t_{i}, j_{i}\right)\right\}_{i=1}^{\infty}$ such that $\left(t_{i}, j_{i}\right) \in \operatorname{dom} x, t_{i}+j_{i} \rightarrow \infty$ and $\mathcal{A}_{\left[t_{i}, j_{i}\right]}^{\Delta} \stackrel{\text { gph }}{\longrightarrow} \varphi$ as $i \rightarrow \infty$. Define $x_{i}(t, j):=x\left(t+t_{i}, j+j_{i}\right)$ for all $(t, j)$ such that $\left(t+t_{i}, j+j_{i}\right) \in \operatorname{dom} x$. It follows that $\mathcal{A}_{[0,0]}^{\Delta} x_{i}=\mathcal{A}_{\left[t_{i}, j_{i}\right]}^{\Delta} \stackrel{\mathrm{gph}}{\longrightarrow} \varphi$ as $i \rightarrow \infty$. Since $x_{i}$ is a bounded sequence, it can be assumed, without loss of generality, that $x_{i} \stackrel{\text { gph }}{\longrightarrow} z$ as $i \rightarrow \infty$. Otherwise, we can always extract a graphically convergent subsequence of $x_{i}$ (see [5, Theorem 6.1]). It follows from nominal well-posedness of $\mathcal{H}_{\mathcal{M}}^{\Delta}$ that $z \in \mathcal{S}_{\mathcal{H}_{\mathcal{M}}^{\Delta}}$ with $\mathcal{A}_{[0,0]}^{\Delta} z=\varphi$. Moreover, we claim that $\mathcal{A}_{[t, j]}^{\Delta} z \in \Omega(x)$ for all $(t, j) \in \operatorname{dom}_{\geq 0}(z)$. To prove this, note from Lemma 2.1 that there exists a sequence of pairs $\left(s_{i}, k_{i}\right)$ such that $\mathcal{A}_{\left[s_{i}, k_{i}\right]}^{\Delta} x_{i} \stackrel{\mathrm{gph}}{\longrightarrow} \mathcal{A}_{[t, j]}^{\Delta} z$ as $i \rightarrow \infty$, which implies $\mathcal{A}_{\left[s_{i}+t_{i}, k_{i}+j_{i}\right]}^{\Delta} \stackrel{\text { gph }}{\longrightarrow} \mathcal{A}_{[t, j]}^{\Delta} z$ as $i \rightarrow \infty$ and hence $\mathcal{A}_{[t, j]}^{\Delta} z \in \Omega(x)$. This shows that $\Omega(x)$ is weakly positively invariant.

Now we prove that $\mathcal{A}_{[t, j]}^{\Delta} \stackrel{\mathrm{gph}}{\longrightarrow} \Omega(x)$ as $t+j \rightarrow \infty$ and $(t, j) \in \mathrm{dom}_{\geq 0}(x)$. Suppose this is not the case. Then there exists some $\varepsilon>0$ and a sequence of pairs $\left(t_{i}, j_{i}\right) \in \operatorname{dom} \geq 0(x)$ such that $\mathbf{d}\left(\mathcal{A}_{\left[t_{i}, j_{i}\right]}^{\Delta} x, \varphi\right) \geq \varepsilon$ for all $\varphi \in \Omega(x)$. Since $\mathcal{A}_{\left[t_{i}, j_{i}\right]}^{\Delta} x$ is a bounded sequence, it can be assumed, without loss of generality, that $\mathcal{A}_{\left[t_{i}, j_{i}\right]}^{\Delta} x \stackrel{\text { gph }}{\longrightarrow} \psi$ as $i \rightarrow \infty$. This shows $\psi \in \Omega(x)$ and contradicts the above inequality. Hence we must have $\mathcal{A}_{[t, j]}^{\Delta} \stackrel{\text { gph }}{\longrightarrow} \Omega(x)$ as $t+j \rightarrow \infty$ and $(t, j) \in \operatorname{dom}_{\geq 0}(x)$.

\section{Invariance principles}

In this section, we prove two invariance principles for hybrid systems with memory, one using a LyapunovKrasovskii (L-K) functional and the other using a Lyapunov-Razumikhin (L-R) function.

The following result utilizes a Lyapunov-Krasovskii (L-K) functional $V$ and proves an invariance principle for hybrid systems with memory, provided that the growth of $V$ along solutions of the hybrid system is bounded by an integral involving a non-positive functional $u_{c}$ and sums involving a non-positive functional $u_{d}$. The result generalizes that for hybrid systems without memory [22] (see also Chapter 8 of [5]) and that for functional differential equations [7].

Theorem 3.1. Let $\mathcal{H}_{\mathcal{M}}^{\Delta}=(\mathcal{C}, \mathcal{F}, \mathcal{D}, \mathcal{G})$ be nominally well-posed. Suppose that there exists a functional $V: \mathcal{M}^{\Delta} \rightarrow$ $\mathbb{R}$ and functionals $u_{c}, u_{d}: \mathcal{M}^{\Delta} \rightarrow \mathbb{R}_{\leq 0}$ such that, for any $x \in \mathcal{S}_{\mathcal{H}_{\mathcal{M}}^{\Delta}}$ and $(\underline{t}, \underline{j}),(\bar{t}, \bar{j}) \in \operatorname{dom}_{\geq 0}(x)$ satisfying $(\underline{t}, j) \preceq(\bar{t}, \bar{j})$ (in lexicographical order),

$$
V\left(\mathcal{A}_{[\bar{t}, j]}^{\Delta} x\right)-V\left(\mathcal{A}_{[\underline{t}, j]}^{\Delta} x\right) \leq \int_{\underline{\underline{t}}}^{\bar{t}} u_{c}\left(\mathcal{A}_{[s, j(s)]}^{\Delta} x\right) d s+\sum_{j=\underline{j}+1}^{\bar{j}} u_{d}\left(\mathcal{A}_{[t(j), j-1]}^{\Delta} x\right),
$$

where $j(s)=\inf \{k:(s, k) \in \operatorname{dom}(x)\}$ and $t(j)=\inf \{s:(s, j) \in \operatorname{dom}(x)\}$. Let $z$ be a bounded and complete solution for $\mathcal{H}_{\mathcal{M}}^{\Delta}$. Then $z$ approaches the largest weakly positively invariant set $\mathcal{R}$ in

$$
\left.V^{-1}(r) \cap \overline{\left(u_{c}^{-1}(0)\right.} \cup u_{d}^{-1}(0)\right) \cap \mathcal{M}_{b, \lambda}^{\Delta}
$$

i.e., $\mathcal{A}_{[t, j]}^{\Delta} z \stackrel{g p h}{\longrightarrow} \mathcal{R}$ as $t+j \rightarrow \infty$ and $(t, j) \in$ dom $_{\geq 0}(z)$, where $r, b, \lambda>0$ are some constants and $\overline{u_{c}^{-1}(0)}$ indicates the closure of $u_{c}^{-1}(0)$ in $\mathcal{M}^{\Delta}$.

Proof. Note from (3.1) that $V\left(\mathcal{A}_{[t, j]}^{\Delta} z\right)$ is non-increasing for $(t, j) \in \operatorname{dom}_{\geq 0}(z)$. Moreover, for all $t+j \geq \Delta+1$ such that $(t, j) \in \operatorname{dom}_{\geq 0}(z)$, we have $\mathcal{A}_{[t, j]}^{\Delta} z \in \mathcal{M}_{b, \lambda}^{\Delta}$ for some fixed $b, \lambda \geq 0$. This is because that $z$ is 
bounded and $\mathcal{F}$ maps each $\mathcal{M}_{b}^{\Delta}$ into a bounded set in $\mathbb{R}^{n}$. Consequently, the set

$$
\left\{A_{[t, j]} z: t+j \geq \Delta+1,(t, j) \in \operatorname{dom}_{\geq 0}(z)\right\}
$$

belongs to the compact set $\mathcal{M}_{b, \lambda}^{\Delta}$. Since $V$ is continuous, $V\left(\mathcal{A}_{[t, j]}^{\Delta} z\right)$ is bounded from below as $t+j \rightarrow \infty$. Therefore, there exists some constant $c$ such that $V\left(\mathcal{A}_{[t, j]}^{\Delta} z\right) \rightarrow c$ as $t+j \rightarrow \infty$ and $(t, j) \in \operatorname{dom}_{\geq 0}(z)$. It follows that $V(\varphi)=c$ for all $\varphi \in \Omega(z)$. Moreover, according to Proposition 2.2, $\Omega(z)$ is weakly positively invariant. Hence, for each $\varphi \in \Omega(z)$, there exists $y \in \mathcal{S}_{\mathcal{H}_{\mathcal{M}}^{\Delta}}$ with $\mathcal{A}_{[0,0]}^{\Delta} y=\varphi$ such that $\mathcal{A}_{[t, j]}^{\Delta} y \in \Omega(z)$ for all $(t, j) \in \operatorname{dom}_{\geq 0}(y)$. We must have $V\left(\mathcal{A}_{[t, j]}^{\Delta} y\right)=c$ for all $(t, j) \in \operatorname{dom}_{\geq 0}(y)$. From (3.1), this implies that, for any $(\underline{t}, \underline{j}),(\bar{t}, \bar{j}) \in \operatorname{dom}_{\geq 0}(x)$ satisfying $(\underline{t}, \underline{j}) \preceq(\bar{t}, \bar{j})$, we have

$$
\int_{\underline{\underline{t}}}^{\bar{t}} u_{c}\left(\mathcal{A}_{[s, j(s)]}^{\Delta} y\right) d s+\sum_{j=\underline{j}+1}^{\bar{j}} u_{d}\left(\mathcal{A}_{[t(j), j-1]}^{\Delta} y\right)=0 .
$$

If $(0,1) \in \operatorname{dom} y$, then the above equality implies that $u_{d}\left(\mathcal{A}_{[0,0]}^{\Delta} y\right)=u_{d}(\varphi)=0$. If $(h, 0) \in \operatorname{dom} y$ for some $h>0$, then the above inequality implies that $\int_{0}^{h} u_{c}\left(\mathcal{A}_{[s, 0]}^{\Delta} y\right) d s=0$. It follows that $u_{c}\left(\mathcal{A}_{[s, 0]}^{\Delta} y\right)=0$ for almost all $s \in[0, h]$. Therefore, $\varphi=\mathcal{A}_{[0,0]}^{\Delta} y \in \overline{u_{c}^{-1}(0)}$.

The following result utilizes a Lyapunov-Razumikhin (L-K) function $V$ and proves another invariance principle for hybrid systems with memory, provided that certain Razumikhin-type conditions on the growth of $V$ are satisfied by solutions of the hybrid system. The result generalizes that for functional differential equations [6] to hybrid systems.

Theorem 3.2. Let $\mathcal{H}_{\mathcal{M}}^{\Delta}=(\mathcal{C}, \mathcal{F}, \mathcal{D}, \mathcal{G})$ be nominally well-posed. Suppose that there exists a continuously differentiable function $V: \mathbb{R}^{n} \rightarrow \mathbb{R}$ such that the following hold:

(i) $\nabla V(\varphi(0,0)) \cdot f \leq 0$ for all $\varphi \in \mathcal{C}$ such that $V(\varphi(0,0)) \geq \bar{V}(\varphi)$ and all $f \in \mathcal{F}(\varphi)$;

(ii) $V(g) \leq \bar{V}(\varphi)$ for all $\varphi \in \mathcal{D}$ and all $g \in \mathcal{G}(\varphi)$,

where $\bar{V}(\varphi):=\max _{-\Delta-1 \leq s+k \leq 0} V(\varphi(s, k))$. Let $z$ be a bounded and complete solution for $\mathcal{H}_{\mathcal{M}}^{\Delta}$. Then $z$ approaches the largest weakly positively invariant set $\mathcal{R}$ in $V^{-1}(r) \cap \mathcal{M}_{b, \lambda}^{\Delta}$, for some constants $r$ and $b, \lambda>0$.

Proof. We aim to prove that $\bar{V}\left(\mathcal{A}_{[t, j]}^{\Delta} z\right)$ is non-increasing for $(t, j) \in \operatorname{dom}_{\geq 0}(z)$. If both $(t, j) \in \operatorname{dom} z$ and $(t, j+1) \in \operatorname{dom} z$, we have $V(z(t, j+1)) \leq \bar{V}\left(\mathcal{A}_{[t, j]}^{\Delta} z\right)$, which implies $\bar{V}\left(\mathcal{A}_{[t, j+1]}^{\Delta} z\right) \leq \bar{V}\left(\mathcal{A}_{[t, j]}^{\Delta} z\right)$. If $(t+s, j) \in$ $\operatorname{dom} x$ for all $s \in[0, h]$ for some small $h>0$, we consider two cases: (a) $V(x(t, j))=\bar{V}\left(\mathcal{A}_{[t, j]}^{\Delta} x\right)$, and (b) $V(x(t, j))<\bar{V}\left(\mathcal{A}_{[t, j]}^{\Delta} x\right)$. Note that, if (a) holds, for each $h^{\prime} \in[0, h]$, there exists $h^{\prime \prime} \in\left[0, h^{\prime}\right]$ such that $V\left(z\left(t+h^{\prime \prime}, j\right)\right)=\bar{V}\left(\mathcal{A}_{\left[t+h^{\prime}, j\right]}^{\Delta} z\right)$. Therefore, we have

$$
\begin{aligned}
D^{+} \bar{V}\left(\mathcal{A}_{[t, j]}^{\Delta} z\right) & :=\limsup _{h^{\prime} \rightarrow 0^{+}} \frac{\bar{V}\left(\mathcal{A}_{\left[t+h^{\prime}, j\right]}^{\Delta} z\right)-\bar{V}\left(\mathcal{A}_{[t, j]}^{\Delta} z\right)}{h^{\prime}} \\
& =\limsup _{h^{\prime} \rightarrow 0^{+}} \frac{V\left(z\left(t+h^{\prime \prime}, j\right)\right)-V(x(t, j))}{h^{\prime}} \\
& \leq \limsup _{h^{\prime \prime} \rightarrow 0^{+}} \frac{V\left(z\left(t+h^{\prime \prime}, j\right)\right)-V(x(t, j))}{h^{\prime \prime}} \\
& \leq 0,
\end{aligned}
$$


where the last inequality is implied by condition (i). If (b) holds, it follows from the continuity of $x(t+s, j)$ on $[0, h]$ that, if $h$ is sufficiently small, then $\bar{V}\left(\mathcal{A}_{[t+s, j]}^{\Delta} x\right) \leq \bar{V}\left(\mathcal{A}_{[t, j]}^{\Delta} x\right)$ for all $s \in[0, h]$. We have proved that $\bar{V}\left(\mathcal{A}_{[t, j]}^{\Delta} x\right)$ is non-increasing for $(t, j) \in \operatorname{dom} x$. The rest of the proof follows exactly from that for Theorem 3.1 with the functional $V$ in Theorem 3.1 replaced by the functional $\bar{V}$ defined here, with $u_{c}=u_{d}=0$.

\section{Stability analysis}

In this section, the invariance principles established in the previous section are applied to the stability analysis of hybrid systems with memory, using L-K functionals and L-R functions, respectively. The notion of stability considered is introduced next.

Definition 4.1. Let $\mathcal{H}_{\mathcal{M}}^{\Delta}$ be a hybrid system with memory and $\mathcal{W} \subseteq \mathbb{R}^{n}$ be a closed set. The set $\mathcal{W}$ is said to be pre-asymptotically stable for $\mathcal{H}_{\mathcal{M}}^{\Delta}$ if the following two hold:

- (stable) for every $\varepsilon>0$, there exists $\delta>0$ such that every solution $x$ to $\mathcal{H}_{\mathcal{M}}^{\Delta}$ with $\left\|\mathcal{A}_{[0,0]}^{\Delta} x\right\|_{\mathcal{W}} \leq \delta$ satifies $|x(t, j)|_{\mathcal{W}} \leq \varepsilon$ for all $(t, j) \in \operatorname{dom} x$, where $|z|_{\mathcal{W}}:=\inf _{y \in \mathcal{W}}|y-z|$ for $z \in \mathbb{R}^{n}$ and $\|\varphi\|_{\mathcal{W}}:=$ $\sup _{\substack{(s, k) \in \operatorname{dom} \varphi \\ s+k \geq-\Delta-1}}|\varphi(s, k)|_{\mathcal{W}}$ for $\varphi \in \mathcal{M}^{\Delta}$;

- (pre-attractive) every complete solution $x$ to $\mathcal{H}_{\mathcal{M}}^{\Delta}$ satisfies $|x(t, j)|_{\mathcal{W}} \rightarrow 0$ as $t+j \rightarrow \infty$ and $(t, j) \in$ $\operatorname{dom} x$.

We start with proving a stability theorem using Lyapunov-Krasovskii functionals. The result has been proved in [17], under slightly different assumptions. Here, we prove it as a corollary of Theorem 3.1.

Theorem 4.1. [17] Let $\mathcal{H}_{\mathcal{M}}^{\Delta}=(\mathcal{C}, \mathcal{F}, \mathcal{D}, \mathcal{G})$ be nominally well-posed and let $\mathcal{W} \subseteq \mathbb{R}^{n}$ be a compact set. Suppose that there exists a functional $V: \mathcal{M}^{\Delta} \rightarrow \mathbb{R}_{\geq 0}$, continuous functions $\hat{u}_{c}, \hat{u}_{d}: \mathbb{R}^{n} \rightarrow \mathbb{R}_{\leq 0}$ that are negative definite with respect to the set $\mathcal{W}$ (i.e., $\hat{u}_{c}(x)$ and $\hat{u}_{c}(x)$ being zero if and only if $x \in \mathcal{W} \subseteq \mathbb{R}^{n}$ ), and $\mathcal{K}_{\infty}$ functions $\alpha_{i}$ $(i=1,2)$ such that

(i) $\alpha_{1}\left(|\varphi(0,0)|_{\mathcal{W}}\right) \leq V(\varphi) \leq \alpha_{2}\left(\|\varphi\|_{\mathcal{W}}\right)$ for all $\varphi \in \mathcal{M}^{\Delta}$;

(ii) for any $x \in \mathcal{S}_{\mathcal{H}_{\mathcal{M}}^{\Delta}}$ and $(\underline{t}, \underline{j}),(\bar{t}, \bar{j}) \in \operatorname{dom}_{\geq 0}(x)$ satisfying $(\underline{t}, \underline{j}) \preceq(\bar{t}, \bar{j})$,

$$
V\left(\mathcal{A}_{[\bar{t}, j]}^{\Delta} x\right)-V\left(\mathcal{A}_{[\underline{\underline{t}}, \underline{\underline{j}}]}^{\Delta} x\right) \leq \int_{\underline{\underline{t}}}^{\bar{t}} \hat{u}_{c}(x(s, j(s))) d s+\sum_{j=j+1}^{\bar{j}} \hat{u}_{d}(x(t(j), j-1)),
$$

where $j(s)=\inf \{k:(s, k) \in \operatorname{dom}(x)\}$ and $t(j)=\inf \{s:(s, j) \in \operatorname{dom}(x)\}$.

Then $\mathcal{W}$ is pre-asymptotically stable for $\mathcal{H}_{\mathcal{M}}$.

Proof. It follows from (4.1) that $V\left(\mathcal{A}_{[t, j]}^{\Delta} x\right)$ is non-increasing for $(t, j) \in \operatorname{dom}_{\geq 0}(x)$, which together with condition (i), implies uniform stability. Since $\mathcal{W}$ is a compact set, this also implies that all solutions are bounded. From Theorem 3.1, any bounded complete solution approaches (in graphical convergence sense) the largest weakly positively invariant set $\mathcal{R}$ in $V^{-1}(c)$ for some $c \geq 0$. If $c=0$, then pre-asymptotic stability follows from (i). In fact, uniform convergence of complete solutions to the compact set $\mathcal{W}$ follows from the fact that they graphically approach $\mathcal{R}$ and (i).

Now we show that $c=0$. Suppose $c>0$ and take any $x \in \mathcal{S}_{\mathcal{H}_{\mathcal{M}}^{\Delta}}$. Then it must be that there exists $\left(t^{*}, j^{*}\right)$ sufficiently large such that $\left|x\left(t^{*}, j^{*}\right)\right|_{\mathcal{W}}>0$. Otherwise, condition (i) would imply that $V\left(\mathcal{A}_{[t, j]}^{\Delta} x\right)=0$ for all sufficiently large $(t, j) \in \operatorname{dom} x$, which would contradict with the assumption $c>0$. It follows from (4.1) that there exists $\left(t^{\prime}, j^{\prime}\right)$ such that $\left(t^{*}, j^{*}\right) \preceq\left(t^{\prime}, j^{\prime}\right)$ such that $V\left(\mathcal{A}_{\left[t^{\prime}, j^{\prime}\right]}^{\Delta} x\right)<V\left(\mathcal{A}_{\left[t^{*}, j^{*}\right]}^{\Delta} x\right)$. Note that both $\mathcal{A}_{\left[t^{\prime}, j^{\prime}\right]}^{\Delta} x \in \mathcal{R}$ and $\mathcal{A}_{\left[t^{*}, j^{*}\right]}^{\Delta} x \in \mathcal{R}$. This contradicts with the fact that $\mathcal{R} \subseteq V^{-1}(c)$ for some $c$. 
The next stability result is based on a Lyapunov-Razumikhin (L-K) function. Again, the result has been proved in [17] and we prove it as a corollary of Theorem 3.2.

Theorem 4.2. [17] Let $\mathcal{H}_{\mathcal{M}}^{\Delta}=(\mathcal{C}, \mathcal{F}, \mathcal{D}, \mathcal{G})$ be nominally well-posed and let $\mathcal{W} \subseteq \mathbb{R}^{n}$ be a compact set. If there exists a continuously differentiable function $V: \mathbb{R}^{n} \rightarrow \mathbb{R}_{\geq 0}, \mathcal{K}_{\infty}$ functions $\alpha_{i}(i=1,2)$, a positive definite and continuous function $\alpha_{3}: \mathbb{R}_{\geq 0} \rightarrow \mathbb{R}_{\geq 0}$, and continuous functions $p: \mathbb{R}_{\geq 0} \rightarrow \mathbb{R}_{\geq 0}$ and $\rho: \mathbb{R}_{\geq 0} \rightarrow \mathbb{R}_{\geq 0}$ with $p(s)>s$ and $\rho(s)<s$ for all $s>0$ such that the following hold:

(i) $\alpha_{1}\left(|\varphi(0,0)|_{\mathcal{W}}\right) \leq V(\varphi(0,0)) \leq \alpha_{2}\left(|\varphi(0,0)|_{\mathcal{W}}\right)$ for all $\varphi \in \mathcal{M}^{\Delta}$;

(ii) $\nabla V(\varphi(0,0)) \cdot f \leq-\alpha_{3}(V(\varphi(0,0)))$ for all $\varphi \in \mathcal{C}$ such that $p(V(\varphi(0,0))) \geq \bar{V}(\varphi)$ and all $f \in \mathcal{F}(\varphi)$;

(iii) $V(g) \leq \rho(\bar{V}(\varphi))$ for all $\varphi \in \mathcal{D}$ and all $g \in \mathcal{G}(\varphi)$,

where $\bar{V}(\varphi):=\max _{-\Delta-1 \leq s+k \leq 0} V(\varphi(s, k))$, then $\mathcal{W}$ is pre-asymptotically stable for $\mathcal{H}_{\mathcal{M}}^{\Delta}$.

Proof. Given any $x \in \mathcal{S}_{\mathcal{H}_{\mathcal{M}}^{\Delta}}$, it follows from the same argument as in the proof of Theorem 3.2 that $\bar{V}\left(\mathcal{A}_{[t, j]}^{\Delta} x\right)$ is non-increasing for $(t, j) \in \mathrm{dom}_{\geq 0}(x)$, which together with condition (i), implies uniform stability. Since $\mathcal{W}$ is a compact set, this also implies that all solutions are bounded. From Theorem 3.2, any bounded complete solution approaches (in graphical convergence sense) the largest weakly positively invariant set $\mathcal{R}$ in $\bar{V}^{-1}(c)$ for some $c \geq 0$. We We claim that

$$
\mathcal{R}=\left\{\varphi \in \mathcal{M}_{b, \lambda}^{\Delta}:|\varphi(s, k)|_{\mathcal{W}}=0, \forall(s, k) \in \operatorname{dom} \varphi\right\}
$$

Suppose that $\mathcal{R}$ contains some $\varphi$ with $\|\varphi\|_{\mathcal{W}}>0$. Since $\mathcal{R}$ is weakly forward invariant, there exists some $z \in \mathcal{S}_{\mathcal{H}_{\mathcal{M}}^{\Delta}}$ such that $\mathcal{A}_{[0,0]}^{\Delta} z=\varphi$ and $\mathcal{A}_{[t, j]}^{\Delta} z \in \mathcal{R}$ for all $(t, j) \in \operatorname{dom}_{\geq 0}(z)$. In particular, this implies that $\bar{V}\left(\mathcal{A}_{[t, j]}^{\Delta} z\right)=\bar{V}\left(\mathcal{A}_{[0,0]}^{\Delta} z\right)=\bar{V}(\varphi)>0$. Pick $\left(t^{*}, j^{*}\right) \in \operatorname{dom} z$ sufficiently large such that $V\left(z\left(t^{*}, j^{*}\right)\right)=$ $\bar{V}\left(\mathcal{A}_{\left[t^{*}, j^{*}\right]^{\prime}}^{\Delta} z\right)=\bar{V}(\varphi)$. In view of condition (iii), it must be that $t^{*} \in \operatorname{int}\left(I_{j^{*}}\right)$. Thus, there exists some $h>0$ such that $\left(s, j^{*}\right) \in \operatorname{dom} z$ for all $s \in\left[t^{*}-h, t^{*}\right]$. Using continuity of $p\left(V\left(z\left(s, j^{*}\right)\right)\right)$ with respect to $s \in\left[t^{*}-h, t^{*}\right]$, there exists $h_{1} \in(0, h)$ such that $p\left(V\left(z\left(s, j^{*}\right)\right)\right)>\bar{V}(\varphi)=\bar{V}\left(\mathcal{A}_{\left[s, j^{*}\right]}^{\Delta} z\right)$ for all $s \in\left[t^{*}-h_{1}, t^{*}\right]$. Therefore, according to condition (ii), $\frac{d V\left(z\left(s, j^{*}\right)\right)}{d s}<0$ for almost all $s \in\left[t^{*}-h_{1}, t^{*}\right]$. This would imply that $V\left(t^{*}, j^{*}\right)<V\left(t^{*}-h_{1}, j^{*}\right)$ and contradicts the definition of $V\left(t^{*}, j^{*}\right)$. We have proved (4.2) and preasymptotic stability follows.

\section{An example}

We use a simple example to illustrate the stability analysis of hybrid systems using invariance principles.

Example 5.1. Consider the following hybrid system:

$$
\begin{aligned}
& \left\{\begin{array}{l}
\dot{z}=f_{p}\left(z, z\left(t-r_{f}\right)\right) \\
\dot{p}=0 \\
\dot{\tau}=1
\end{array}\right\} \quad z \in \mathbb{R}^{n}, p \in \mathcal{P}, \tau \in[0, \delta], \\
& \left\{\begin{array}{l}
z^{+}=g_{p}\left(z, z\left(t-r_{g}\right)\right) \\
p^{+} \in \mathcal{P} \\
\tau^{+}=0
\end{array}\right\} \quad z \in \mathbb{R}^{n}, p \in \mathcal{P}, \tau \in\{\delta\},
\end{aligned}
$$

where $z \in \mathbb{R}^{n}$ is the ordinary state, $p \in \mathcal{P}$ is the mode, $\tau \in \mathbb{R}_{\geq 0}$ is the state of a timer, $r_{f}>0$ and $r_{g}>0$ are constant delays, and $\delta>0$ is a fixed period for mode switches and state jumps. 
Let $\psi=(\varphi, p, \tau) \in \mathcal{M}^{\Delta}$. One way of interpreting the hybrid system above is to use the following data:

$$
\begin{gathered}
\mathcal{F}=\left[\begin{array}{c}
\bigcup_{\left(-r_{f}, k\right) \in \operatorname{dom} \psi} f_{p}\left(\varphi(0,0), \varphi\left(-r_{f}, k\right)\right) \\
0 \\
1
\end{array}\right] \\
\mathcal{G}=\left[\begin{array}{c}
\bigcup_{\left(-r_{g}, k\right) \in \operatorname{dom} \psi} g_{p}\left(\varphi(0,0), \varphi\left(-r_{g}, k\right)\right) \\
\mathcal{P} \\
0
\end{array}\right] \\
\mathcal{C}=\left\{\psi=(\varphi, p, \tau) \in \mathcal{M}^{\Delta}: \tau(0,0) \subseteq[0, \delta]\right\} . \\
\mathcal{D}=\left\{\psi=(\varphi, p, \tau) \in \mathcal{M}^{\Delta}: \tau(0,0)=\delta\right\} . \\
\mathcal{W}=\{0\} \times \mathcal{P} \times[0, \delta] \subseteq \mathbb{R}^{n} \times \mathcal{P} \times \mathbb{R}_{\geq 0} .
\end{gathered}
$$

Clearly, $|\psi(0,0)|_{\mathcal{W}}=|\varphi(0,0)|$ and $\|\psi\|_{\mathcal{W}}=\|\varphi\|$ for all $\psi=(\varphi, p, \tau) \in \mathcal{M}^{\Delta}$, where $\|\varphi\|=\sup _{\substack{(t, j) \in \operatorname{edom} \varphi \\-\Delta-1 \leq t+j \leq 0}}|\varphi(t, j)|$.

Let $x=(z, p, \tau) \in \mathbb{R}^{n+2}$ and consider a Lyapunov function candidate of the form $V(x):=U_{p}(z) e^{-\sigma \tau}$, where $\sigma$ is a constant to be determined and, for $p \in \mathcal{P}$, each $U_{p}: \mathbb{R}^{n} \rightarrow \mathbb{R}^{+}$satisfies

(i) $\alpha_{1}(|z|) \leq U_{p}(z) \leq \alpha_{2}(|z|)$ for all $z \in \mathbb{R}^{n}$ and $p \in \mathcal{P}$;

(ii) there exist constants $q$ and $\hat{q}>0$ such that $\nabla U_{p}(z) \cdot f_{p}(z, \hat{z}) \leq q U_{p}(z)+\hat{q} U_{p}(\hat{z})$ for all $(z, \hat{z}) \in \mathbb{R}^{2 n}$ and $p \in \mathcal{P}$;

(iii) there exist constants $\rho>0$ and $\hat{\rho}>0$ such that $U_{p}\left(g_{p}(z, \hat{z})\right) \leq \rho U_{p}(z)+\hat{\rho} U_{p}(\hat{z})$ for all $(z, \hat{z}) \in \mathbb{R}^{2 n}$ and $p \in \mathcal{P}$.

Now we verify that conditions of Theorem 4.2 hold. First, for $\psi=(\varphi, p, \tau) \in \mathcal{M}^{\Delta}$,

$$
\begin{aligned}
\alpha_{1}\left(|\psi(0,0)|_{W}\right) e^{-\delta|\sigma|} & \leq V(\psi(0,0))=U_{p}(\varphi(0,0)) e^{-\sigma \tau} \\
& \leq \alpha_{2}\left(|\psi(0,0)|_{W}\right) e^{\delta|\sigma|} .
\end{aligned}
$$

Hence, condition (i) is verified. Second,

$$
\begin{aligned}
& \nabla V(\psi(0,0)) \cdot f \\
& \quad=\left[\nabla U_{p}(\varphi(0,0)) e^{-\sigma \tau}, 0,-\sigma U_{p}(\varphi(0,0)) e^{-\sigma \tau}\right] \cdot\left[\begin{array}{c}
f_{c} \\
0 \\
1
\end{array}\right] \\
& \quad=e^{-\sigma \tau}\left(\nabla U_{p}(\varphi(0,0)) \cdot f_{c}-\sigma U_{p}(\varphi(0,0))\right),
\end{aligned}
$$

where $f=\left[\begin{array}{lll}f_{c} & 0 & 1\end{array}\right]^{\prime} \in \mathcal{F}(\varphi)$ and hence $f_{c}=f_{p}\left(\varphi(0,0), \varphi\left(-r_{f}, k\right)\right)$ for some $\left(-r_{f}, k\right) \in \operatorname{dom} \psi$. It follows from condition (ii) above on $U_{p}$ that

$$
\begin{aligned}
\nabla V(\psi(0,0)) \cdot f \\
\leq e^{-\sigma \tau}\left(q U_{p}(\varphi(0,0))+\hat{q} U_{p}\left(\varphi\left(-r_{f}, k\right)\right)-\sigma U_{p}(\varphi(0,0))\right) \\
\leq-\sigma U_{p}(\varphi(0,0)) e^{-\sigma \tau}+q U_{p}(\varphi(0,0)) e^{-\sigma \tau} \\
\quad+\hat{q} \mu U_{p\left(-r_{f}, k\right)}\left(\varphi\left(-r_{f}, k\right)\right) e^{-\sigma \tau\left(-r_{f}, k\right)} e^{|\sigma| \delta} \\
\leq-\sigma V(\psi(0,0))+\hat{q} \mu e^{|\sigma| \delta} U_{\left.p\left(-r_{f}, k\right)\right)}\left(\varphi\left(-r_{f}, k\right)\right) e^{\left.-\sigma \tau\left(-r_{f}, k\right)\right)} \\
\leq(-\sigma+q) V(\psi(0,0))+\bar{q} \bar{V}_{[0,0]}(\psi)
\end{aligned}
$$


where $\bar{q}=\hat{q} \mu e^{|\sigma| \delta}, \mu \geq 1$ is such that $U_{p}(z) \leq \mu U_{q}(z)$ for all $z \in \mathbb{R}^{n}$, and $\bar{V}_{[0,0]}(\psi)=\max _{-\Delta \leq s+k \leq 0} V(\psi(s, k))$, provided that $-\Delta \leq-r_{f}+k \leq 0$ whenever $\left(-r_{f}, k\right) \in \operatorname{dom} \psi$. Hence, condition (ii) of Theorem 4.2 is verified if $\sigma-q>\bar{q}$. Finally, for $g=\left(g_{d}, p, 0\right) \in \mathcal{G}(\psi), V(g)=U_{p}\left(g_{d}\right)$, where

$$
g_{d} \in \bigcup_{\left(-r_{g}, k\right) \in \operatorname{dom} \psi} g_{p}\left(\varphi(0,0), \varphi\left(-r_{g}, k\right)\right) .
$$

It follows from condition (iii) above on $U_{p}$ that

$$
\begin{aligned}
V(g)= & U_{p}\left(g_{d}\right) \leq \rho U_{p}(\varphi(0,0))+\hat{\rho} U_{p}\left(\varphi\left(-r_{g}, k\right)\right) \\
\leq & \rho e^{\sigma \delta} U_{p}(\varphi(0,0)) e^{-\sigma \delta} \\
& \left.\quad+\hat{\rho} \mu e^{\max (\sigma, 0) \delta} U_{p\left(-r_{g}, k\right)}\left(\varphi\left(-r_{g}, k\right)\right)\right) e^{-\sigma \tau\left(-r_{g}, k\right)} \\
\leq & \bar{\rho} \bar{V}_{[0,0]}(\psi),
\end{aligned}
$$

for some $\left(-r_{g}, k\right) \in \operatorname{dom} \psi$, where $\bar{\rho}=\rho e^{\sigma \delta}+\hat{\rho} \mu e^{\max (\sigma, 0) \delta}$. Hence, condition (iii) of Theorem 4.2 is verified if $\bar{\rho}<1$. Thus, the set $\mathcal{W}$ is pre-asymptotically stable for the hybrid system defined above, if

$$
\sigma-q>\bar{q}=\hat{q} \mu e^{|\sigma| \delta} \text { and } \bar{\rho}=\rho e^{\sigma \delta}+\hat{\rho} \mu e^{\max (\sigma, 0) \delta}<1
$$

hold simultaneously.

Remark 5.1. In particular, the analysis above applies to following two cases:

(i) $q+\hat{q}<0, \rho+\hat{\rho} \mu>1$, and $\hat{\rho} \mu<1$. This may correspond to the case where the dynamics during flow are stable, whereas the jump dynamics are not. The conditions in (5.3) can always be satisfied by choosing $\sigma \in(q+\hat{q}, 0)$ and $\delta$ sufficiently large;

(ii) $q+\hat{q}>0$ and $\rho+\hat{\rho} \mu<1$. This may correspond to the case where the dynamics during flow are unstable, whereas the jump dynamics are stable. The conditions in (5.3) can always be satisfied by choosing $\sigma>q+\hat{q} \mu>0$ and $\delta$ sufficiently small.

\section{Conclusions}

In this paper, we proved two invariance principles for hybrid systems with memory, which are formulated using hybrid functional inclusions. An important step in establishing such invariance principles is to prove certain invariance properties for the limit set of hybrid memory arcs resulted from a bounded and complete hybrid trajectory. It is shown that such invariance properties can be shown in terms of graphical convergence. Applications of the invariance principles are demonstrated on the stability analysis of hybrid systems memory, where we provided alternative proofs for two recent stability results for such systems. A simple example is used to illustrate how these results can be applied.

\section{Acknowledgements}

This work is supported, in part, by Royal Society grant IE130106, EU FP7 grant PCIG13-GA-2013-617377, US AFOSR grants FA9550-12-1-0127, US NSF grant ECCS-1232035, and US AFOSR grant FA9550-15-1-0155. 


\section{References}

[1] Bacciotti, A., Mazzi, L., 2005. An invariance principle for nonlinear switched systems. Systems \& Control Letters 54 (11), 1109-1119.

[2] Chellaboina, V., Bhat, S. P., Haddad, W. M., 2003. An invariance principle for nonlinear hybrid and impulsive dynamical systems. Nonlinear Analysis: Theory, Methods \& Applications 53 (3), 527-550.

[3] Chen, W.-H., Zheng, W. X., 2009. Input-to-state stability and integral input-to-state stability of nonlinear impulsive systems with delays. Automatica 45 (6), 1481-1488.

[4] Goebel, R., Sanfelice, R. G., Teel, A. R., 2008. Invariance principles for switching systems via hybrid systems techniques. Systems \& Control Letters 57 (12), 980-986.

[5] Goebel, R., Sanfelice, R. G., Teel, A. R., 2012. Hybrid Dynamical Systems: Modeling, Stability, and Robustness. Princeton University Press.

[6] Haddock, J., Terjéki, J., 1983. Liapunov-Razumikhin functions and an invariance principle for functional differential equations. Journal of differential equations 48 (1), 95-122.

[7] Hale, J. K., 1965. Sufficient conditions for stability and instability of autonomous functional-differential equations. Journal of Differential Equations 1 (4), 452-482.

[8] Hespanha, J. P., 2004. Uniform stability of switched linear systems: extensions of lasalle's invariance principle. Automatic Control, IEEE Transactions on 49 (4), 470-482.

[9] LaSalle, J., 1960. The extent of asymptotic stability. Proceedings of the National Academy of Sciences of the United States of America 46 (3), 363.

[10] LaSalle, J., 1960. Some extensions of Liapunov's second method. Circuit Theory, IRE Transactions on 7 (4), 520-527.

[11] Liu, J., Liu, X., Xie, W.-C., 2009. Invariance principles for impulsive switched systems. Dynamics of Continuous, Discrete \& Impulsive Systems. Series B. Applications \& Algorithms 16, 631-654.

[12] Liu, J., Liu, X., Xie, W.-C., 2011. Generalized invariance principles for switched delay systems. IMA J. Math. Control Inform. 28, 19-39.

[13] Liu, J., Liu, X., Xie, W.-C., 2011. Input-to-state stability of impulsive and switching hybrid systems with time-delay. Automatica 47, 899-908.

[14] Liu, J., Teel, A. R., 2012. Generalized solutions to hybrid systems with delays. In: Proc. of IEEE Conference on Decision and Control (CDC). pp. 6169-6174.

[15] Liu, J., Teel, A. R., 2014. Hybrid systems with memory: existence and well-posedness of generalized solutions. SIAM J. on Control and Optimization, submitted.

[16] Liu, J., Teel, A. R., 2014. Hybrid systems with memory: modelling and stability analysis via generalized solutions. In: Proc. of IFAC World Congress. pp. 6019-6024.

[17] Liu, J., Teel, A. R., 2014. Lyapunov-based sufficient conditions for stability of hybrid systems with memory. IEEE Trans. Automat. Control, submitted.

[18] Liu, X., Ballinger, G., 2001. Uniform asymptotic stability of impulsive delay differential equations. Comput. Math. Appl. 41, 903-915. 
[19] Liu, X., Shen, J., 2006. Stability theory of hybrid dynamical systems with time delay. IEEE Trans. Automat. Control 51, 620-625.

[20] Mancilla-Aguilar, J. L., García, R. A., 2006. An extension of LaSalle's invariance principle for switched systems. Systems \& Control Letters 55 (5), 376-384.

[21] Rockafellar, R. T., Wets, R. J. B., 1998. Variational Analysis. Vol. 317. Springer-Verlag.

[22] Sanfelice, R. G., Goebel, R., Teel, A. R., 2007. Invariance principles for hybrid systems with connections to detectability and asymptotic stability. Automatic Control, IEEE Transactions on 52 (12), 2282-2297.

[23] Wang, J., Cheng, D., Hu, X., 2009. An extension of LaSalle's invariance principle for a class of switched linear systems. Systems \& Control Letters 58 (10), 754-758.

[24] Yan, P., Özbay, H., 2008. Stability analysis of switched time delay systems. SIAM J. Control Optim. 47, 936-949.

[25] Yuan, R., Jing, Z., Chen, L., 2003. Uniform asymptotic stability of hybrid dynamical systems with delay. IEEE Trans. Automat. Control 48, 344-348.

[26] Zhang, Y., Wang, M., Xu, H., Teo, K. L., 2014. Global stabilization of switched control systems with time delay. Nonlinear Analysis: Hybrid Systems 14, 86-98. 
LaTeX Souce Files
Click here to download LaTeX Souce Files: hybrid_invariance_R1.tex

LaTeX Souce Files
Click here to download LaTeX Souce Files: hybrid_invariance_R1.tex

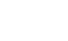

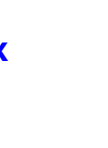

$\left(\frac{10}{2}\right.$

(1)
(1) (1) (1)

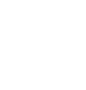
(1)

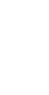
(1)

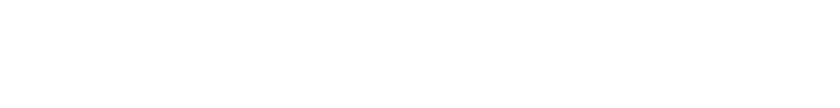
(1) (1) . . . . . . . . . . . . . 
LaTeX Souce Files (.bib file)
Click here to download LaTeX Souce Files: hybrid_invariance.bib

LaTeX Souce Files (.bib file)
Click here to download LaTeX Souce Files: hybrid_invariance.bib

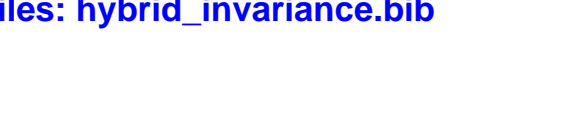

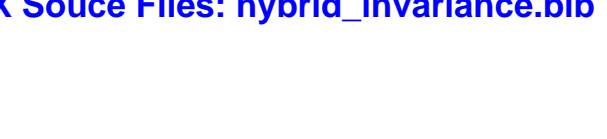

-

$\sqrt{10}$

$\sqrt{10}$

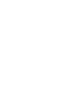

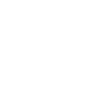
(1) $\sqrt{2}$ (1) (1) -

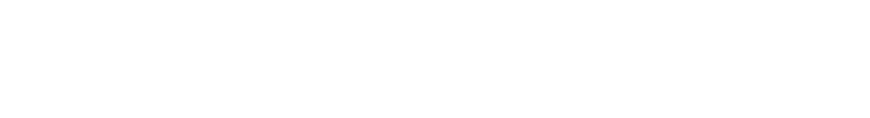
.

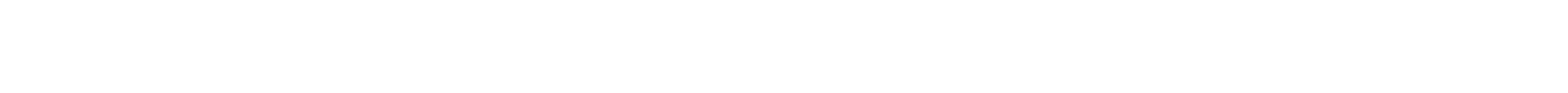
-

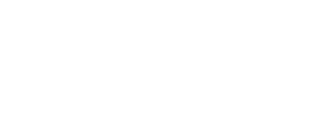

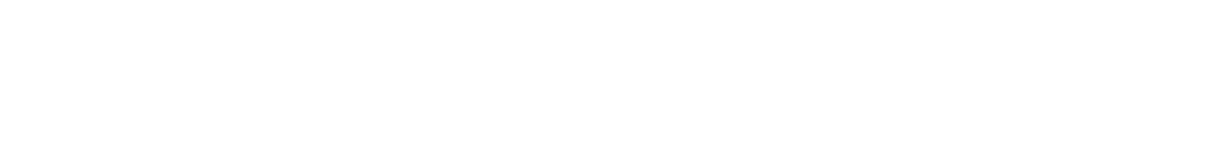
(1)

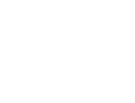
(1)

(1)
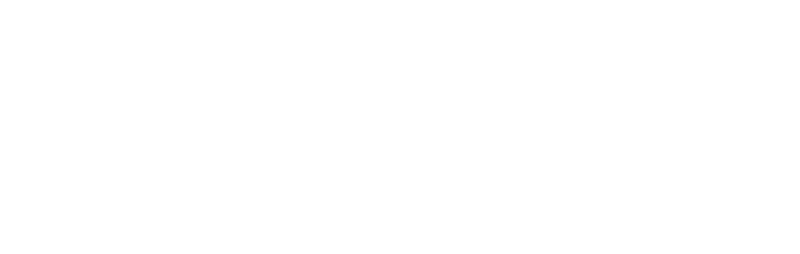